\title{
An Enhanced Security-Constrained Unit Commitment Model with Reserve Response Set Policies
}

\author{
Nan Li, Nikita G. Singhal, and Kory W. Hedman \\ Arizona State University \\ \{nanli4, nsinghal, kory.hedman\}@asu.edu
}

\begin{abstract}
Security-constrained unit commitment (SCUC) is a classical problem used for day-ahead commitment, dispatch, and reserve scheduling. Even though SCUC models acquire reserves, $N-1$ reliability is not guaranteed. This paper presents an enhanced securityconstrained unit commitment formulation that facilitates the integration of stochastic resources and accounts for reserve deliverability issues. In this formulation, the SCUC is modified to incorporate a reserve response set model. The enhanced reserve model aims to predict the effects of nodal reserve deployment on critical transmission lines so as to improve the deliverability of reserves postcontingency. The enhanced reserve policies are developed using a knowledge discovery process as a means to predict reserve activation. The approach, thus, aims to acquire reserve at prime locations that face fewer reserve deliverability issues. The results show that the proposed approach consistently outperforms contemporary approaches. All numerical results are based on the IEEE 73-bus test case.
\end{abstract}

\section{Nomenclature}

Indices

$c \quad$ Contingency.

$g \quad$ Generator.

$k \quad$ Reserve zone.

$l \quad$ Transmission line.

$n \quad$ Bus location.

$t \quad$ Time period.

$z(c) \quad$ Reserve zone where contingency $c$ is located.

Parameters

$c_{g}() \quad$ Variable cost function; generator $g$.

$c_{g}^{N L} \quad$ No-load cost; generator $g$.

$c_{g}^{\text {res }} \quad$ Reserve cost; generator $g$.

$c_{g}^{S U} \quad$ Start-up cost; generator $g$.

$d_{n t} \quad$ Demand; bus $n$, time $t$.

$D T_{g} \quad$ Minimum down time; generator $g$.

\begin{tabular}{|c|c|}
\hline$F_{l t}^{\text {RateA }}$ & $\begin{array}{l}\text { Capacity (Rate A); transmission line } l \text {, time } \\
t \text {. }\end{array}$ \\
\hline$F_{l t}^{\text {RateC }}$ & $\begin{array}{l}\text { Emergency capacity (Rate C); transmission } \\
\text { line } l \text {, time } t \text {. }\end{array}$ \\
\hline$G^{C}$ & Set for critical generators. \\
\hline$L^{C}$ & Set for critical transmission paths. \\
\hline$P_{g}^{\min }$ & Minimum capacity; generator $g$. \\
\hline$P_{g}^{\max }$ & Maximum capacity; generator $g$. \\
\hline$P T D F_{n, l}$ & Power transfer distribution factor. \\
\hline$R_{g}^{10}$ & 10 minute ramp up/down rate; generator $g$. \\
\hline$R_{g}^{60}$ & 60 minute ramp up/down rate; generator $g$. \\
\hline$U T_{g}$ & Minimum up time; generator $g$. \\
\hline$\alpha$ & Choice of reserve sharing policy. \\
\hline$\Gamma_{g t}^{C},\left(\overline{\Gamma_{g t}^{C}}\right)$ & $\begin{array}{l}\text { Reserve activation factor; generator } g \text {, } \\
\text { contingency } c \text {, time } t \text { (determined from } \\
\text { training phase). }\end{array}$ \\
\hline \multicolumn{2}{|l|}{ Variables } \\
\hline$F_{l t}$ & Power flow on line $l$; time $t$. \\
\hline$i_{n t}$ & Power injection; bus $n$, time $t$. \\
\hline$P_{g t}$ & Real power production; generator $g$, time $t$. \\
\hline$r_{g t}$ & Reserve; generator $g$, time $t$. \\
\hline$r_{g t}^{c},\left(r_{g, s, t}^{c}\right)$ & $\begin{array}{l}\text { Activated reserve; generator } g \text {, contingency } \\
c \text {, time } t \text { (net load scenario } s \text { ). }\end{array}$ \\
\hline$\tilde{r}_{k t}^{c}$ & Reserve in zone $k$; contingency $c$, time $t$. \\
\hline$S_{k t}^{Z(c)}$ & $\begin{array}{l}\text { Reserve sharing limit from zone } k \text { to } \\
\text { contingency zone } c \text { time } t \text {. }\end{array}$ \\
\hline$u_{g t}$ & $\begin{array}{l}\text { Unit commitment variable ( } 0 \text { offline, } 1 \\
\text { online); generator } g \text {, period } t \text {. }\end{array}$ \\
\hline$v_{g t}$ & $\begin{array}{l}\text { Startup variable ( } 1 \text { for startup, } 0 \text { otherwise); } \\
\text { generator } g \text {, period } t \text {. }\end{array}$ \\
\hline$w_{g t}$ & $\begin{array}{l}\text { Shutdown variable ( } 1 \text { for shutdown, } 0 \\
\text { otherwise); generator } g \text {, period } t \text {. }\end{array}$ \\
\hline
\end{tabular}

\section{Introduction}

The Federal Energy Regulatory Commission (FERC) defines ancillary services as those services "necessary to support the transmission of electric power from seller to purchaser, given the obligations of control areas and transmitting utilities within those control areas, to maintain reliable operations of the 
interconnected transmission system," [1]. Therefore, to ensure a continuous and reliable supply of electricity, electricity markets acquire reserves to protect against unexpected events. Reserve is defined as backup capability that provides flexibility to satisfy energy imbalances and mitigate uncertainty. In this paper, reserve can come from many different resources including, but not limited to, generators, demand response, and storage. The $N-1$ reliability criterion, set by the North American Electric Reliability Corporation (NERC), requires the system to recover from the loss of any single bulk element without involuntary load shedding. Since contemporary mathematical programs, used within market management systems, rely on proxies for the $N$-1 requirement, the market solutions may procure reserves that are not deliverable. With the integration of stochastic resources (e.g., wind and solar), it is becoming more challenging to maintain system reliability at least cost.

Various approaches have been developed and used to address uncertainty. An example of one such approach is the use of deterministic models [2]-[5]. In [2]-[5], reserve policies are modeled as pre-defined system-wide reserve requirements and included as constraints. However, a system-wide reserve constraint only imposes a quantity requirement; it does not impose a requirement on the location of the reserves. In order to improve the deliverability of reserve, zonal reserve models are developed in [6]-[10]. Instead of purely using system-wide reserve requirements, predefined regional or zonal reserve requirements are utilized, in [6]-[10], to address the allocation of reserves. The motivation behind using reserve zones is to disperse the reserve across the system such that the deliverability of reserves is improved.

While zonal reserve models can improve the deliverability of reserve, they still do not guarantee reserve deliverability for all $N-1$ events. Intra-zonal congestion that is not acknowledged, along with interzonal congestion, can prevent needed reserves from reaching the desired locations. Thus, to overcome the shortcomings of deterministic models, stochastic programming has been proposed. In [11]-[14], uncertainties are explicitly represented in the model and are solved simultaneously. By explicitly formulating the network constraints, the reserves are ensured to be deliverable for the events explicitly modeled. Since uncertainties are explicitly represented in the model, no pre-defined reserve requirements or reserve zones are needed. However, while more robust solutions can be obtained from stochastic programming models, stochastic programs are less computationally tractable when compared to deterministic models.

In this work, enhanced reserve policies are proposed to improve the deliverability of reserve in a power system with stochastic resources. Specifically, the proposed reserve policies are targeted at improving the deliverability of contingency reserves in the postcontingency state, i.e., ensuring that the reserve is deliverable after a contingency has occurred in the system. In this paper, we focus on generator contingencies; line contingencies are not addressed as existing security-constrained unit commitment (SCUC) models already include transmission contingencies.

The primary contributions of the paper are as follows: 1) developed enhanced reserve policies to improve system reliability and market efficiency, 2) designed dynamic reserve policies that acknowledge system operating conditions, and 3) developed a methodology that utilizes offline knowledge discovery processes on historical data or leverage Monte-Carlo simulations that generate hypothetical data.

The remainder of the paper is organized as follows. Section 2 describes the existing industry practices used to handle large-scale complex models. Section 3 discusses contemporary industry-based policy-driven approaches that are currently embedded within SCUC models to ensure sufficient reserves. Section 4 discusses the proposed reserve model. Section 5 presents the numerical results and Section 6 concludes this paper along with a discussion on future work.

\section{Industry practices}

As per the reliability standards set by NERC, the system is required to be able to withstand an $\mathrm{N}-1$ event. In other words, given a system with $N$ elements, operators are required to continue serving demand reliably following the failure of any single bulk power system element. With such reliability requirements, the ideal approach would be to model all $N-1$ events explicitly within SCUC. However, it is a challenge to model the full network model for large-scale power systems for both pre- and post-contingency states and have it solved within the required time window. As reported in [15], the Midcontinent Independent System Operator (MISO) manages a system with about 45,000 buses. In order to meet the market clearing time requirements, MISO employs a 1200 seconds time limit and a $0.1 \%$ MIP relative gap for the day-ahead (DA) SCUC. As stated in [15], MISO constantly encounters performance challenges in solving its DA SCUC model with the aforementioned limits; thus, expanding the model in order to explicitly represent contingency scenarios within the model would be computationally burdensome. Therefore, in order to address the performance challenges that arise from large-scale systems, the ISOs in the United States currently rely on heuristics, approximations, and policies rather than solving stochastic programs. 
The California ISO (CAISO) utilizes dynamic operating transfer capability and nomograms in order to improve dispatch while maintaining system reliability. A nomogram is a set of operating or scheduling rules that are used to approximate reliability limits [16]. The central idea behind using nomograms is to operate the system, defined by the nomogram, such that no thermal, voltage or stability limits are violated following the occurrence of a contingency.

Nomograms are generally determined through an offline analysis stage using prior operating experience with the system and by predicting future operating conditions for the system. The primary motivation for using such nomograms is to satisfy multiple operating limits simultaneously without having to represent the corresponding constraints in the model explicitly.

Another popular practice used by the industry is the reduction of the power transfer distribution factor (PTDF) matrix by omitting PTDFs, which have an absolute value less than a cutoff value. CAISO uses a 2\% cut-off while MISO and the Pennsylvania-New Jersey-Maryland (PJM) Interconnection employ a 5\% PTDF cutoff threshold [17]-[19]. A simple yet neat heuristic policy like this can help reduce the computational complexity drastically.

It is important to note that the increased reliance on heuristics, approximations, and policies in order to reduce the computational burden of models is not new. In fact, all mixed-integer linear programs (MILPs) for power systems are approximations. The direct-current optimal power flow (OPF) problem is a linear approximation of the non-linear alternating-current OPF problem. The commonly used UC formulation is a natural evolution of approximations and heuristics that have been present in the literature for many years.

In recent years, stochastic programming [11]-[14] has been widely proposed as a solution to address system uncertainty. However, it is challenging to solve a large-scale stochastic program within required timeframes. There are additional challenges to adopting stochastic programming. These issues include, but are not restricted to, the lack of transparency for stakeholders, the consistency of solution quality subject to the solution timeframe, the selection of the uncertainties to be modeled, and market pricing issues. Approximations remain a necessary approach to help strike a balance between model complexity and model accuracy.

\section{Contemporary reserve policies}

In order to improve the deliverability of reserves in large-scale power systems while maintaining the computational tractability of the model, one potential solution is to utilize policy-driven approaches to provide enhancement to the existing UC and economic dispatch models. In [20], a policy is described as a rule (or a function) that determines a decision given the available information in a particular state. Today, most of the existing reserve rules involve some sort of approximations. The $N-1$ deterministic criterion, which requires the total system reserve to be sufficient to cover any single generator contingency, is a policy. This policy is equivalent to a single-zone model and is described by (1). An example of another such policy or criterion is to require the system-wide reserves to be greater than or equal to a fraction, $\eta$, of the demand.

$\sum_{g} r_{g t} \geq P_{g t}+r_{g t}, \forall g \in G, t \in T$.

$\sum_{g} r_{g t} \geq \eta \% \sum_{n} d_{n t}, \forall t \in T$

Enforcing reserve requirements on a zonal basis rather than a system-wide basis is a policy choice. Today, most of the ISOs use reserve zones to ensure that sufficient reserve is held within import-constrained areas. A simplistic representation of one such reserve model [21], which is an extension of the reserve model used by ISO New England [6], is given below:

$\sum_{k \in Z} \tilde{r}_{k t}^{c} \geq P_{c t}+r_{c t}, \forall c \in G, t \in T$
$\tilde{r}_{k t}^{c} \leq \sum_{g \in G(k)} r_{g t}, \forall c \in G, k \in Z, t \in T$
$\tilde{r}_{k t}^{c} \leq S_{k t}^{Z(c)}, \forall c \in G, k \in Z, t \in T$
$S_{k t}^{Z(c)}=\alpha\left(F_{l t_{k-z(c)}}^{\text {Ratec }}\right) \pm F_{l t_{k-Z(c)}}, \forall c \in G, k \in Z, t \in T$

Here, $\tilde{r}_{k t}^{c}$ signifies reserve in zone $k$ that is categorized as deliverable to contingency zone $c$ in time $t$. Equation (3) requires the sum of the imported reserves to be sufficient to replace the underlying generator contingency, (4) requires the amount of exported reserves to be less than the net reserves that are held within the zone, and (5) sets a limit on the amount of reserves (imported/exported) that can be shared between adjacent zones. The maximum amount of reserves that can be shared between two zones is equivalent to the available transfer capability on the inter-zonal links. In other words, it is equal to the difference between the emergency line rating (Rate $\mathrm{C}$ ) of the inter-zonal link and the corresponding flow on the link. In this case, ' $\alpha$ ' is equal to one and such a policy would be considered as a less conservative policy when compared to ' $\alpha$ ' equal to less than one. For the purpose of this model, a smaller value of ' $\alpha$ ' indicates a more conservative reserve policy whereas a larger value of ' $\alpha$ ' indicates a less conservative reserve policy. In this model, the reserve sharing limit $\left({ }^{\prime} \alpha^{\prime}\right)$ may be pre-determined based on an offline analysis and is system-dependent. In the latter half of this paper, this particular reserve model will be referred to as the base-case model and is used to compare the relative performance of the proposed reserve model. 
For policy-driven approaches, one common characteristic is that such approaches almost always entail some approximations and attempt to utilize the knowledge that is gained offline during a prior stage in order to improve the complicated decision-making process. In [10], dynamic reserve zones are developed to improve the deliverability of reserves. As the level of penetration of renewables increases, traditional static zones will no longer be able to adequately reflect the changing operating conditions in the system. Therefore, zone partitioning techniques are developed in [10] to change the partitioning of the zones in the system on a daily or an hourly basis. The partitioning is based on information that is obtained from an offline analysis stage. By analyzing historical data or performing Monte-Carlo simulations during the offline analysis stage, statistical information about the flow on each line can be obtained, which is then used to determine the partition of the zones in the system.

In [22], post-contingency zonal reserve deployment transmission constraints are employed to improve the transfer of reserves between different zones. The authors in [22] utilize a zonal reserve model and formulate the effect of reserve deployment on the flows on critical inter-zonal transmission lines in the system explicitly. By incorporating the postcontingency zonal reserve deployment transmission constraints into the model, the zonal reserve quantity requirement is implicitly determined by the model rather than being provided as an input or a predetermined policy. By testing the approach on MISO's system, the authors in [22] show that the use of zonal reserve deployment transmission constraints can effectively improve the deliverability of reserve while keeping the computational time manageable. A similar approach can also be found in [23]. Thus, it is important to note that effectively and efficiently allocating reserves across the system is a trending area of research. This paper seeks to demonstrate and compare the performance of different reserve policies in ensuring efficient and reliable operations of the grid.

\section{Proposed reserve model: methodology and mathematical formulations}

The proposed reserve model aims to achieve two primary goals: 1) address the reserve quantity and location issues to improve reserve deliverability in the post-contingency state and 2) minimize the added computational difficulty for the SCUC model and maintain the scalability of the model.

Figure 1 illustrates the overall process of the proposed approach. The proposed approach consists of two primary phases. The first phase is the offline training phase, which involves a knowledge discovery process. In the training phase, a modified SCUC, which includes a contingency-based reserve model, is solved to determine the quantity and location of reserves for each generator contingency. The modified SCUC solved during the offline training phase will be referred to as the training phase SCUC from hereon. This is followed by a reserve deliverability check in order to investigate the deliverability of the reserve that each generator provides for each contingency and each net load scenario. The next step in phase one is to reflect the deliverability of the reserve provided by each generator by updating a parameter $\Gamma_{g t}^{c}$. This process is repeated until a termination criterion is met.

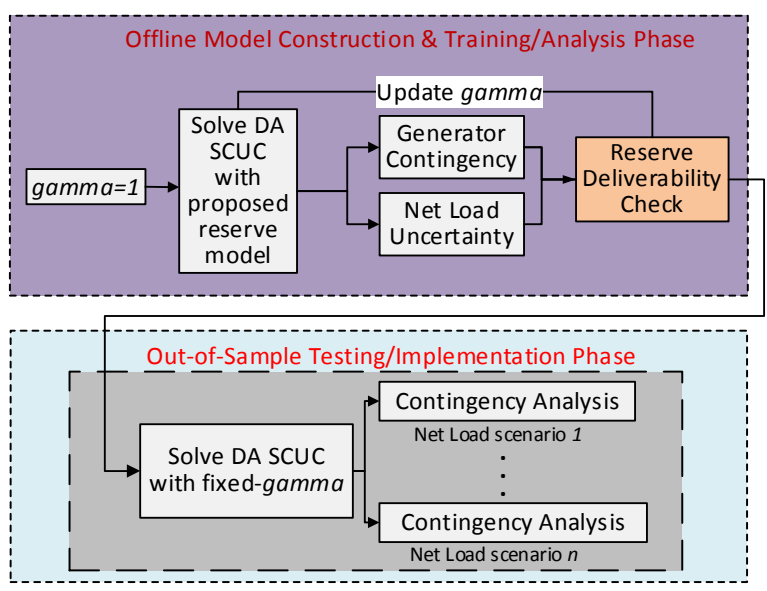

Figure 1. Flowchart for the proposed approach.

The second phase of the proposed approach is the testing phase, which represents the DA stage. In the testing phase, an enhanced SCUC model, which incorporates a responsive reserve model (presented in section 4.3) is solved. The parameter $\Gamma_{g t}^{c}$ is obtained from the offline training phase and is utilized in the testing phase SCUC to allocate reserves (provided by each generator) at prime locations (i.e., the locations that deliver reserves in the post-contingency state) in the system. Again, this step is followed by contingency analysis to test the DA market solution from SCUC against generator contingencies combined with net load scenarios in the system. The proposed SCUC and reserve models used in each stage are described in more detail in the following sub-sections.

\subsection{Day-ahead SCUC formulation with a single zone reserve model}

The DA SCUC model is formulated as a MILP. The objective (7) is to minimize the total system operating cost, which includes both the fuel costs and the cost of reserves. A single-zone reserve model, which is also 
referred to as a myopic reserve policy, is implemented. The complete SCUC formulation is shown in (7)-(23).

$$
\begin{aligned}
& \text { Min: } \sum_{\forall t, \forall g} c_{g}\left(P_{g t}\right)+c_{g}^{N L} u_{g t}+c_{g}^{S U} v_{g t}+c_{g}^{r e s} r_{g t} \\
& \text { Subject to: } \\
& i_{n t}=\sum_{\forall g(n)} P_{g t}-d_{n t}, \forall n, t \\
& \sum_{\forall n} i_{n t}=0, \forall t \\
& F_{l t}=\sum_{\forall n} P T D F_{n, l} i_{n t}, \forall l, t \\
& -F_{l t}^{\text {Rate } A} \leq F_{l t} \leq F_{l t}^{R a t e}, \forall l, t \\
& P_{g t}+r_{g t} \leq P_{g}^{\text {max }} u_{g t}, \forall g, t \\
& P_{g}^{\text {min }} u_{g t} \leq P_{g t}, \forall g, t \\
& \sum_{q=t-U T_{g}+1}^{t} v_{g q} \leq u_{g t}, \forall g, t \in\left\{U T_{g}, \ldots, T\right\} \\
& \sum_{q=t-D T_{g}+1}^{t} w_{g q} \leq 1-u_{g t}, \forall g, t \in\left\{D T_{g}, \ldots, T\right\} \\
& v_{g t}-w_{g t}=u_{g t}-u_{g, t-1}, \forall g, t \\
& r_{g t} \leq R_{g}^{10} u_{g t}, \forall g, t \\
& P_{g, t}-P_{g, t-1} \leq R_{g}^{60} u_{g, t-1}+R_{g}^{S U} v_{g t}, \forall g, t \\
& P_{g, t-1}-P_{g, t} \leq R_{g}^{60} u_{g t}+R_{g}^{S D} w_{g t}, \forall g, t \\
& \sum_{g} r_{g t} \geq P_{g t}+r_{g t}, \forall g, t \\
& \sum_{g} r_{g t} \geq \eta \% \sum_{n} d_{n t}, \forall t \\
& u_{g t} \in\{0,1\}, \forall g, t \\
& 0 \leq v_{g t}, w_{g t} \leq 1, \forall g, t
\end{aligned}
$$

In the above formulation, (8) models the power injection at every bus and (9) guarantees the balance between load and generation across the system. Constraint (10) represents the dc power flow on each line and (11) imposes the transmission line limits. Constraints (12) and (13) represent the generator output limit constraints. The minimum up and down time constraints are shown in (14)-(16). Constraint (17) represents the ramp rate restriction for spinning reserves. In this paper, contingency reserves are modeled by spinning reserves and are used to mitigate the contingencies in the system. The hourly ramp rate constraints are shown in (18) and (19). System-wide spinning reserve requirements are modeled in (20) and (21). Constraints (20) and (21) together require that the system-wide reserve be no less than the single largest generator contingency or $\eta \%$ of the total demand in the system, whichever is greater. In this paper, only spinning reserve is modeled. However, the model can be extended to account for other reserve types.

\subsection{A contingency-based reserve model for the offline training stage}

During the offline knowledge discovery stage, the objective is to identify the prime locations where reserves are deliverable post-contingency and determine the appropriate quantity of reserves that each generator should provide. Since the system operating condition changes post-contingency, it is important to capture the deviation in the line flows from the precontingency state. To achieve this goal, postcontingency line flow constraints for critical paths, similar to those utilized in [22] and [23], are included in the model. The contingency-based reserve model for the offline training stage is described in (24)-(29).

$$
\begin{aligned}
& \sum_{g} r_{g t} \geq P_{g t}+r_{g t}, \forall g, t \\
& \sum_{g} r_{g t} \geq \eta \% \sum_{n} d_{n t}, \forall t \\
& \sum_{g} r_{g t}^{c} \geq P_{c t}+r_{c t}, \forall c \in G, t \\
& r_{g t}^{c} \leq \Gamma_{g t}^{c} r_{g t}, \forall c \in G, g, t \\
& \quad-F_{l t}^{\text {RateC }} \leq F_{l t}-P_{c t} P T D F_{n(c), l}+\sum_{g} P T D F_{n(g), l} r_{g t}^{c} \\
& \quad \leq F_{l t}^{\text {RateC }}, \forall c \in G^{C}, l \in L^{C}, t \\
& \Gamma_{g t}^{c} \in[0,1], \forall c \in G, g, t
\end{aligned}
$$

In the above reserve model, (24) and (25) are similar to (20) and (21), which represent the systemwide reserve requirement. Constraint (26) is the contingency-based reserve requirement, which ensures that the sum of deployed reserves in each contingency event covers the loss of the corresponding generation. Variable $r_{g t}^{c}$ represents the activated reserve for each contingency while $r_{g t}$ indicates the available reserve. Constraint (27) indicates that the activated reserve for each contingency should be less than or equal to a fraction $\left(\Gamma_{g t}^{c}\right)$ of the scheduled reserve. In (27), the parameter $\Gamma_{g t}^{c}$ is the reserve activation coefficient taking on values between zero and one. In the offline training stage, $\Gamma_{g t}^{c}$ is initially set to one and is then updated iteratively to reflect the deliverability of the reserve from each generator in each contingency event until a certain criterion is met.

Constraint (28) represents the post-contingency line flow constraints. In (28), $F_{l t}$ is the pre-contingency line flow on line $l$, which is computed using (10). The second component, $P_{c t} P T D F_{n(c), l}$, represents the change in the flow on line $l$ due to the loss of generator $c$. The third component $\sum_{g} P T D F_{n(g), l} r_{g t}^{c}$ models the effect of reserve deployment on line $l$ in contingency $c$. Constraint (28) captures the effects of reserve deployment on critical transmission paths and models the line flows in the post-contingency state explicitly. Here, critical transmission paths refer to the set of lines that are frequently congested in the pre- or postcontingency states, which can then cause reserve deliverability issues. Critical paths can be preidentified based on historical data, operational procedures, and offline studies [22]. In (28), set $G^{C}$ indicates the critical generator set. Therefore, (28) is only formulated for the largest generators and the critical transmission paths. Usually, loss of a larger generating unit results in more post-contingency violations when compared to the loss of a smaller generating unit due to the increased quantity of 
deployed reserves and the larger deviation in the line flows on critical transmission paths. However, this is not always the case and it is system-dependent based on the location of the activated reserves; thus, set $G^{C}$ can be extended to include smaller generating units as well. In this case study, set $G^{C}$ was pre-defined to include only the larger units after weighing the tradeoff between model accuracy and model complexity.

\subsection{Reserve response set model for the testing stage}

In the testing stage, the DA SCUC model is solved with a responsive reserve model that includes reserve response set policies. Here, the parameter $\overline{\Gamma_{g t}^{c}}$, obtained from the offline training stage, is embedded within the reserve model. The complete formulation of the responsive reserve model is described in (30)-(33).

$\sum_{g} r_{g t} \geq \eta \% \sum_{n} d_{n t}, \forall t$

$\sum_{g} \bar{\Gamma}_{g t}^{c} r_{g t} \geq P_{c t}+r_{c t}, \forall c \in G, t$

$-F_{l t}^{\text {RateC }} \leq F_{l t}-P_{c t} P T D F_{n(c), l}+\sum_{g} P T D F_{n(g), l} \overline{\Gamma_{g t}^{c}} r_{g t}$

$\leq F_{l t}^{\text {RateC }}, \forall c \in G^{C}, l \in L^{C}, t$

$\overline{\Gamma_{g t}^{c}} \in[0,1], \forall c \in G, g, t$

Constraint (30) is the same as (21), which requires the system-wide reserve to be greater or equal to a fraction, $\eta$, of the demand. Constraint (31) represents the contingency-based reserve requirement. The parameter $\overline{\Gamma_{g t}^{c}}$ is obtained from the offline training stage and reflects the fraction of the reserve $r_{g t}$ that is potentially deliverable in contingency $c$. By incorporating the deliverability information into the reserve model, (31) determines a response set for each contingency event. The response sets defined by (31) identify the potential deliverable locations as well as the quantity of reserves in each contingency event. Constraint (32) is similar to (28), which models the post-contingency line flows and aims to ensure that reserves can be transferred through critical transmission paths when deployed. In the testing stage, the post-contingency line flow constraints are formulated for the same set of critical transmission paths and the same set critical generators that were used in the training phase.

\subsection{Updating parameter $\Gamma_{g t}^{c}$}

During the offline knowledge discovery phase, data generated by Monte-Carlo simulations is analyzed to determine the quantity of reserves that are activated from each generator and identify the prime locations for reserves. In other words, the offline knowledge discovery process is used to determine a response set for each contingency. The response set is identified using the parameter $\Gamma_{g t}^{c}$, which again aims to capture the deliverability of reserve at each location in each contingency. This sub-section describes the iterative approach that is used to update $\Gamma_{g t}^{c}$.

After the training phase SCUC is solved; its solution is tested against generator contingencies combined with net load uncertainty to analyze reserve deliverability issues in each scenario. Here, each scenario refers to the combination of a particular realization of a net load scenario in a particular generator contingency state. In this study, 100 different net load scenarios were utilized in the reserve deliverability check stage of the training phase. Once the reserve deliverability check is completed, the reserve deployed $\left(r_{g, s, t}^{c}\right)$, for contingency $c$ and net load scenario $s$, can be obtained. For each generator, contingency scenario and time period, the amount of reserve deployed for the different net load scenarios are then sorted in the descending order following which a particular value of $r_{g, s, t}^{c}$ (the deployed reserve) is chosen based on the choice of a pre-determined exceedance level. This value of $r_{g, s, t}^{c}$ is then utilized in updating the parameter $\Gamma_{g t}^{c}$ using (34). The exceedance level represents a measure of the fraction of times that an event exceeds a particular reference level. An illustration of the exceedance level is presented in Figure 2.

$\Gamma_{g t}^{c}=\frac{r_{g, s, t}^{c}}{r_{g t}}$

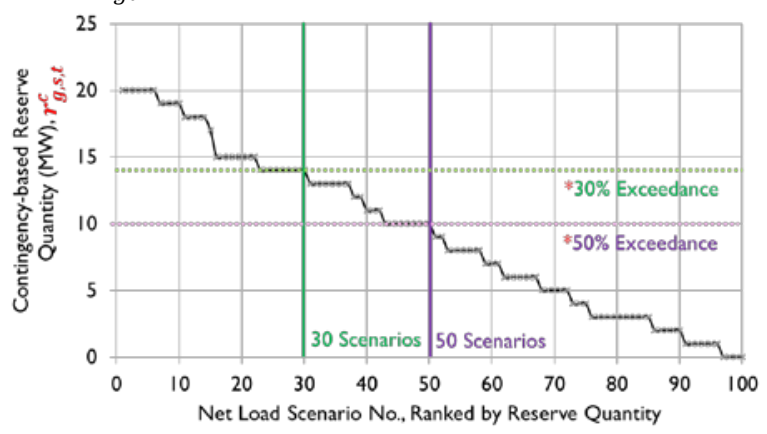

Figure 2. Illustration of exceedance level.

In Figure 2, if a 50\% exceedance level is chosen as a reference, then $r_{g, s, t}^{c}$ will take on the value of $10 \mathrm{MW}$. This exceedance level implies that, for the 100 net load scenarios tested for contingency $c$, generator $g$, and period $t, 50 \%$ of the time $r_{g, s, t}^{c}$ is larger than $10 \mathrm{MW}$. A lower exceedance level represents a less conservative reserve policy; the resultant $\Gamma_{g t}^{c}$ is larger stating that a larger fraction of reserve from generator $g$ is deliverable in contingency $c$ and period $t$. Similarly, a higher exceedance level represents a more conservative reserve policy, which results in a smaller $\Gamma_{g t}^{c}$ to indicate 
that only a small fraction of reserve from generator $g$ can be delivered in contingency $c$ and period $t$.

\section{Case Study}

The proposed methodology was implemented and evaluated on the reliability test system-1996 (RTS-96) [24]. The system contains 73 buses, 117 transmission lines, and 96 conventional generators with a total generation capacity of 10215 MW. A piecewise linear cost function was used to represent the variable fuel costs. The system was partitioned into two zones using the zone partitioning technique presented in [10]. The resulting zone partition is shown in Figure 3 . In the test system, zone one consists of nodes \#1 to \#48 and zone two consists of nodes \#49 to \#73. The line capacity of the two inter-zonal links, which includes lines 73-21 and 66-47, are reduced to create congestion. The two highlighted lines (lines with red stars) in Figure 3 represent the critical paths that are modeled in the proposed reserve model. It is important to note that the critical transmission path set can be extended to include intra-zonal links; for this study, only interzonal links are considered. The critical generator set $G^{C}$ includes $350 \mathrm{MW}$ generators or larger.

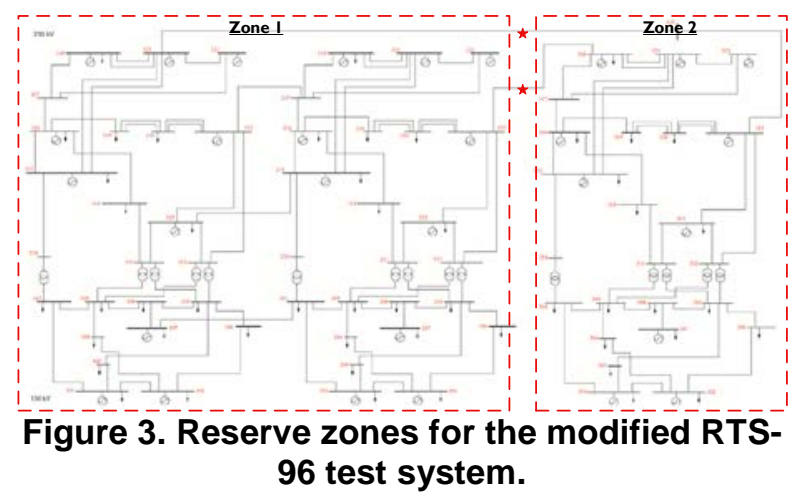

In order to test for the robustness of the proposed approach, the proposed methodology was implemented and evaluated on three different test days (with varying load profiles) during which the base-case peak load reached 8550 MW on each of the three test days. Subsequently, in the contingency analysis, 100 different net load scenarios were utilized in the out-ofsample testing phase. It is assumed that the net load at each bus in the system follows a Gaussian distribution with zero mean. The variance of the Gaussian distribution was selected such that the resultant uncertainty is about $7 \%$. Note that the distribution of the net load at each bus may not necessarily be Gaussian. The assumption of a Gaussian distribution in the case study is to approximate the net load without overcomplicating the scenario generation process. More accurate distributions can be adopted in future work. Monte-Carlo simulation was performed to generate the required 100 scenarios used in the case study. The proposed algorithm was written in Java and solved with CPLEX version 12.6.0. All simulations were run on a computer with the following specifications: Intel ${ }^{\circledR}$ Xeon ${ }^{\circledR}$ CPU X5687 @ 3.60 GHz, 48 GB RAM, and 64-bit operating system. The relative MIP gap was set to 0.002 (or $0.2 \%$ ). Since the majority of the post-contingency congestion occurred on line 66-47, the post-contingency line flow constraint was formulated only on line 66-47, which is one of the highlighted lines in Figure 3.

The numerical results in the sub-sections below show the results for out-of-sample testing on three different test days on the modified IEEE 73-bus test system at one-hour resolution. The relative performance of the base-case (i.e., the reserve model with varying ' $\alpha$ ' policies) and the proposed reserve models are compared based on an analysis of: 1) the DA SCUC cost versus the expected security violations, 2) the number of security violations versus the size of the expected security violations, 3) computational time, and 4) the cost savings obtained with the proposed approach by varying the penalty price for security violations.

\subsection{Testing using scenarios from day one}

This section presents the results when comparing the proposed approach to the base-case approach, for one sample test day. The base-case approach refers to the ' $\alpha$ ' policy approach presented in section 3 where the reserves are modeled using (3)-(6).

A sensitivity analysis was performed in order to set the termination criteria for $\Gamma_{g t}^{c}$. For the purpose of selecting the termination criteria, the offline model construction and training phase was implemented with three different choices of the number of iterations to be performed. In other words, for simulations performed on day one, the number of iterations was set to one, three, and five respectively. Figure 4 shows a distribution of the DA SCUC costs versus the expected security violations obtained with the base-case approach (with varying ' $\alpha$ ' policies) and the proposed approach (with varying termination criteria). It is pertinent to note that the size of the bubbles in Figure 4 is representative of the number of cases (over all contingencies and time periods) with violations for each net load scenario. 


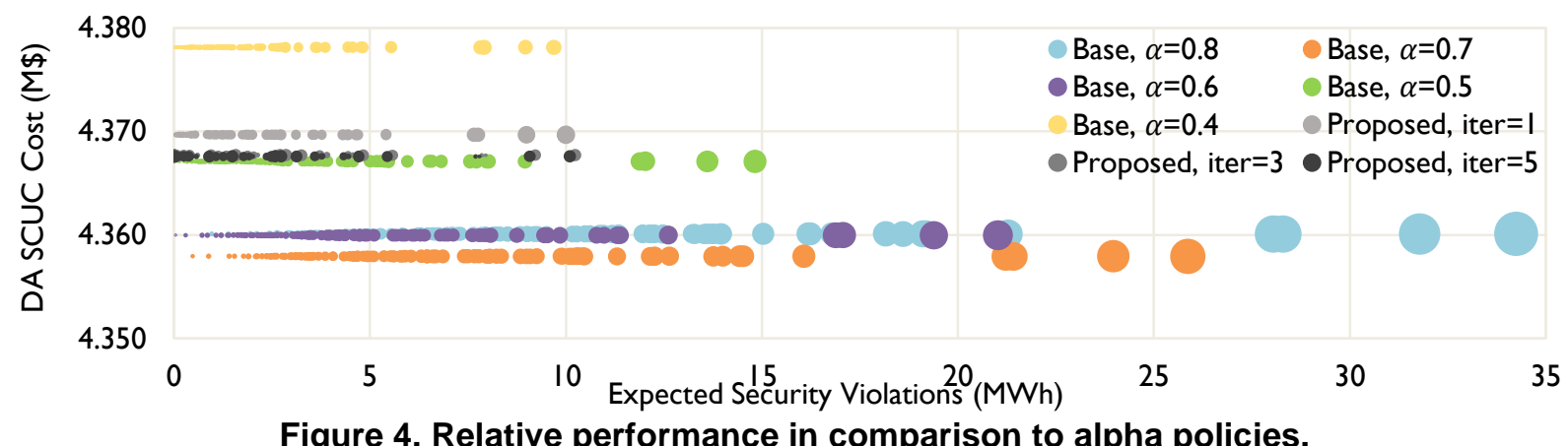

In this case study, since the DA SCUC is solved only once for each tested approach for each day, the DA SCUC cost is the same for all the net load scenarios for each approach. It can be seen that, for the 100 net load scenarios tested in this study, the proposed approach outperforms the ' $\alpha$ ' policy approach when less conservative reserve sharing policies are used. When compared to the ' $\alpha$ ' policy approach with $\alpha$ varying from 0.5 to 0.8 , the proposed approach has lower expected security violations as well as fewer cases with security violations. For the case in which $\alpha$ equals to 0.4 , the proposed methodology has a similar performance in terms of security violations but with reduced DA SCUC costs. Also, since very little improvement was obtained when increasing the number of iterations from three to five, the termination criterion was set to three for the other test days.

Figure 5 shows the average percent cost savings obtained with the proposed approach, with the number of iterations set to three, in comparison to the ' $\alpha$ ' policy approach. The percent cost savings obtained with the proposed approach is directly proportional to the penalty price used for the security violations. Here, the penalty price can be interpreted as an approximation of the cost to correct the unreliable solution out of the market. Future work will involve repeating the process outlined in this paper using an out-of-market correction phase after contingency analysis to obtain the cost to move from an unreliable to a reliable solution.

Figure 6 shows a zoomed-in chart comparing the number of security violations against the expected security violations (in $\mathrm{MWh}$ ) for the DA market solution for each net load scenario for the two reserve models. It can be seen that the proposed approach outperforms the ' $\alpha$ ' policy approach (with respect to both the measures) for most net load scenarios. The proposed approach outperforms the base-case approach with varying ' $\alpha$ ' policies because it more appropriately allocates reserves at prime locations with better potential deliverability while respecting the critical transmission constraints in the post-contingency state.

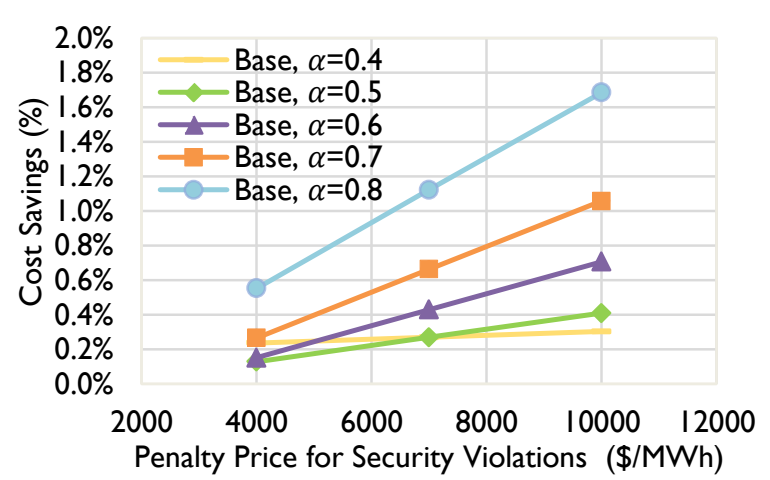

Figure 5. Average percent cost savings in comparison to alpha policies.

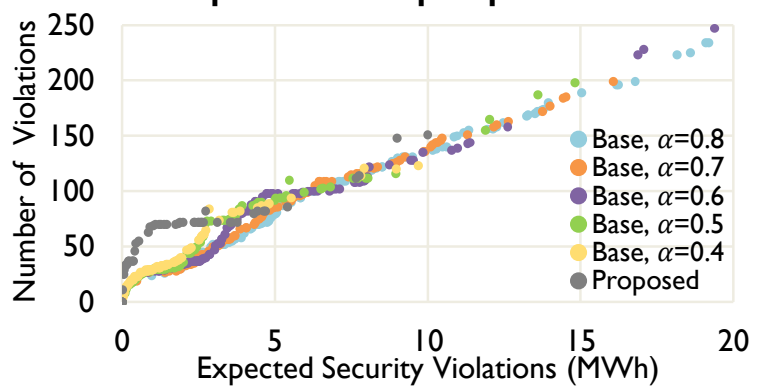

Figure 6 . The number of violations vs. the size of expected security violations.

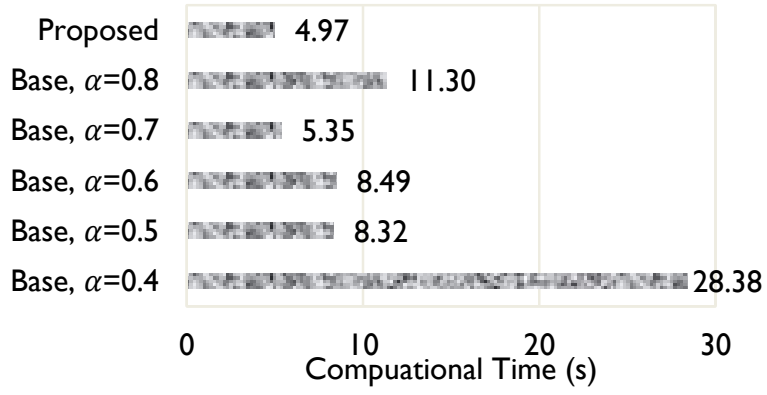

Figure 7. Computational time comparison.

Figure 7 shows a comparison of the computational time (in seconds). The proposed approach is comparable to the base-case approach with varying ' $\alpha$ ' policies and does not add to the computational complexity of contemporary SCUC models. However, it is pertinent to note that, although the proposed 
approach has the shortest solution time, this number can vary (within a reasonable range) depending up on the number of critical lines and generator contingencies that are modeled. As the proposed approach is tested on a relatively small test system, we do not expect the proposed approach to always have a shorter solution time than the ' $\alpha$ ' policy approaches. However, the results demonstrate that the proposed approach can improve the deliverability of reserves while having minimal added computational complexity.

\subsection{Testing using scenarios from different days}

In order to test the robustness of the proposed approach, the model was evaluated on scenarios from two different test days. The results for days: two and three are shown in Figure 8 to Figure 11. It can be seen from the figures that the proposed approach consistently outperforms the base-case approach.

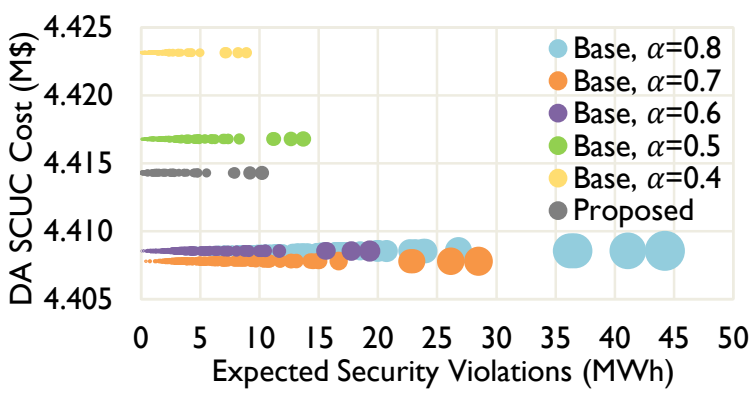

Figure 8. Relative performance in comparison to alpha policies for day two.

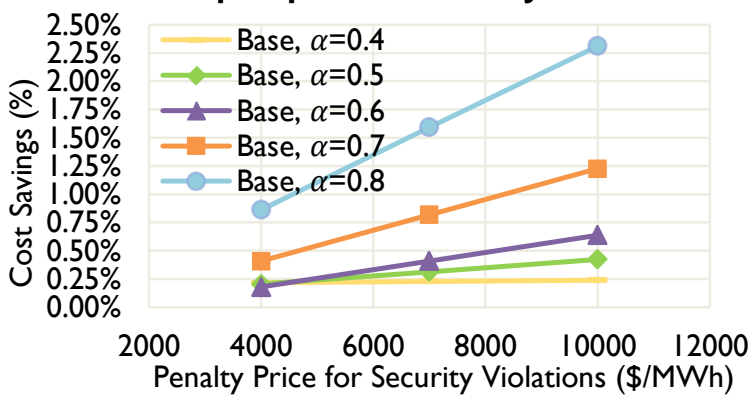

Figure 9. Percent cost savings in comparison to alpha policies for day two.

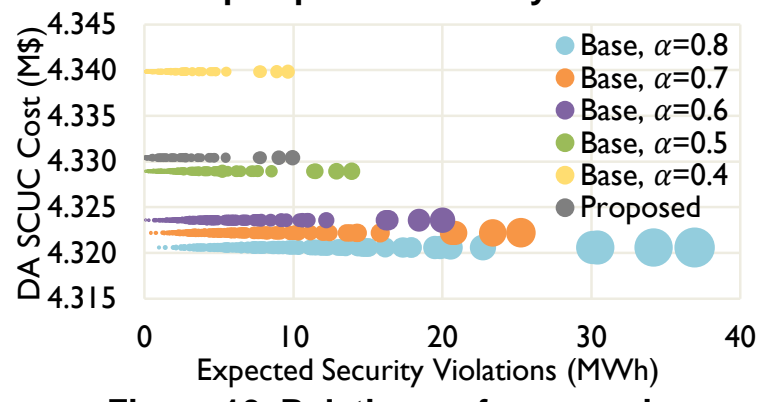

Figure 10. Relative performance in comparison to alpha policies for day three.

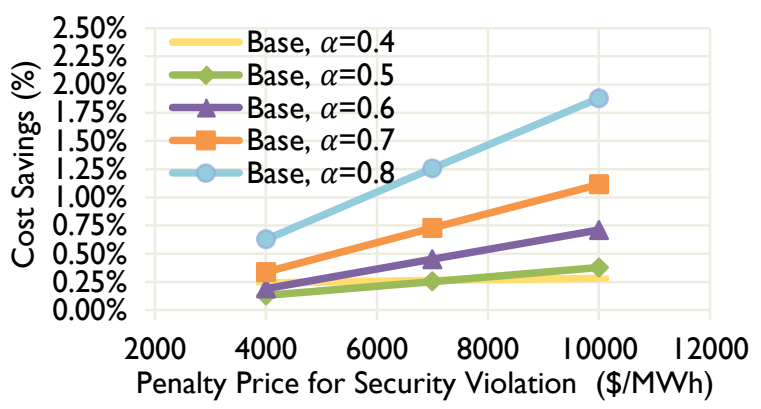

Figure 11. Percent cost savings in comparison to alpha policies for day three.

\section{Conclusions}

In order to deal with the growing concerns over resource uncertainty and variability in contemporary market management systems, smart and well-designed reserve policies, which improve existing deterministic models and facilitate the transition to stochastic programs, are proposed. The proposed offline knowledge discovery approach enhances the reliability of the market solution while also reducing the overall operational costs. The uncertainty and variability associated with stochastic resources and the constantly changing system conditions warrant the utilization of stochastic models when determining reserve requirements. Hence, a Monte-Carlo simulation based approach, which enhances the existing deterministic approaches with minimal added computational burden, is proposed. Additionally, the proposed model appropriately addresses the allocation issues with respect to reserves.

Future work will focus on investigating the market implications and the scalability (i.e., large-scale implementation) of the proposed approach. Future work will also focus on examining hybrid dynamic reserves with stochastic programs. In other words, an investigation on whether such reserve policies can be embedded within stochastic programming algorithms (e.g., progressive hedging, Benders' decomposition) in order to improve convergence and scalability. The primary goal is to develop and evaluate reserve procurement policies that can be applied to the DA or real-time deterministic operations in order to accommodate stochastic operations.

\section{References}

[1] FERC, Guide to Market Oversight - Glossary, 2016. [Online]. Available: http://www.ferc.gov/marketoversight/guide/glossary.asp

[2] D. Gan and E. Litvinov, "Energy and reserve market designs with explicit consideration to lost opportunity cost," 
IEEE Trans. Power Syst., vol. 18, no. 1, pp. 53-59, Feb. 2003.

[3] K. Cheung, P. Shamsollahi, D. Sun, J. Milligan, and M. Potishnak, "Energy and ancillary service dispatch for the interim ISO New England electricity market," IEEE Trans. Power Syst., vol. 15, no. 3, pp. 968-974, Aug. 2000.

[4] M. Madrigal and V. H. Quintana, “A security-constrained energy and spinning reserve markets clearing system using an interior-point method,” IEEE Trans. Power Syst., vol. 15, no. 4, pp. 1410-1416, Nov. 2000.

[5] Z. Li and M. Shahidehpour, "Security-constrained unit commitment for simultaneous clearing of energy and ancillary services markets,” IEEE Trans. Power Syst., vol. 20, no. 2, pp. 1079-1088, May 2005.

[6] T. Zheng and E. Litvinov, "Contingency-based zonal reserve modeling and pricing in a co-optimized energy and reserve market,” IEEE Trans. Power Syst., vol. 23, no. 2, pp. 277-286, May 2008.

[7] T. Wu, M. Rothleder, Z. Alaywan, and A. D. Papalexopoulos, "Pricing energy and ancillary services in integrated market systems by an optimal power flow,” IEEE Trans. Power Syst., vol. 19, no. 1, pp. 339-347, Feb. 2004.

[8] X. Ma, D. Sun, and K. Cheung, "Energy and reserve dispatch in a multi-zone electricity market," IEEE Trans. Power Syst., vol. 14, no. 3, pp. 913-919, Aug. 1999.

[9] T. Alvey, D. Goodwin, X. Ma, D. Streiffert, and D. Sun, "A security-constrained bid-clearing system for the New Zealand wholesale electricity market," IEEE Trans. Power Syst., vol. 13, no. 2, pp. 340-346, May 1998.

[10] F. Wang and K. W. Hedman, "Dynamic reserve zones for day-ahead unit commitment with renewable resources," IEEE Trans. Power Syst., vol. 30, no. 2, pp. 612-620, March 2015.

[11] J. M. Arroyo and F. Galiana, "Energy and reserve pricing in security and network-constrained electricity markets,” IEEE Trans. Power Syst., vol. 20, no. 2, pp. 634643, May 2005.

[12] F. Galiana, O. Bouffard, J. Arroyo, and F. Restrepo, "Scheduling and pricing of coupled energy and primary, secondary, and tertiary reserves,” Proc. of the IEEE, vol. 93, no. 11, pp. 1970-1983, Nov. 2005.

[13] J. Wang, M. Shahidehpour, and Z. Li, "Contingencyconstrained reserve requirements in joint energy and ancillary services auction,” IEEE Trans. Power Syst., vol. 24, no. 3, pp. 1457-1468, Aug. 2009.
[14] A. Papavasiliou, S. S. Oren, and R. P. O'Neill, "Reserve requirements for wind power integration: a scenario-based stochastic programming framework," IEEE Trans. Power Syst., vol. 26, no. 4, pp. 2197-2206, Nov. 2011.

[15] Y. Chen, A. Casto, F. Wang, Q. Wang, X. Wang, and J. Wan, "Improving large scale day-ahead security constrained unit commitment performance,” IEEE Trans. Power Syst., accepted for publication.

[16] Transmission Utilization Group, "COI utilization report," May 2011. [Online]. Available: http://www.oasis.oati.com/WASN/WASNdocs/COI_Utilizati on_Report_S.Anners.pdf

[17] CAISO, Business Practice Manual for Market Operations, Feb 2015. [Online]. Available: https://bpmcm.caiso.com/BPM\%20Document\%20Library/M arket\%20Operations/BPM_for_Market\%20Operations_V43_ redline.pdf

[18] MISO, Interconnection Process Queue Reform, Feb 2016. [Online]. Available: https://www.misoenergy.org/Library/Repository/Meeting\%2 0Material/Stakeholder/IPTF/2016/20160211/20160211\%20I PTF\%20Item\%2004\%20Queue\%20Reform\%20BPM\%20Pre sentation.pdf

[19] MISO and PJM Interconnection LLC, Impact of the PJM 2016/2017 Base Residual Auction on the North Carolina Electric System, Feb 2015. [Online]. Available: https://www.pjm.com/ /media/committeesgroups/committees/teac/20150212/20150212-nc-impacts-of2016-17-bra.ashx

[20] W. B. Powell, Approximate Dynamic Programming: Solving the Curses of Dimensionality, $2^{\text {nd }}$ Ed. New York: Wiley, 2001.

[21] J. D. Lyon, M. Zhang, and K. W. Hedman, "Locational reserve disqualification for distinct scenarios,” IEEE Trans. Power Syst., vol. 30, no. 1, pp. 357-364, Jan. 2015.

[22] Y. Chen, P. Gribik, and J. Gardner, "Incorporating post zonal reserve deployment transmission constraints into energy and ancillary service co-optimization,” IEEE Trans. Power Syst., vol. 29, no. 2, pp. 537-549, Mar. 2014.

[23] R. Ferrero and M. Shahidehpour, "Optimal reserve allocation and pricing," IEEE Power and Energy Society General Meeting, Jul. 2003.

[24] Power System Test Case Archive, Univ. of Washington, Dept. of Elec. Eng., 2010. [Online]. Available: www.ee.washington.edu/research/pstca/index.html 